\section{CD45, une protéine phosphatase cible importante dans le traitement des leucémies aiguës myéloblastiques}

Laetitia Saint-Paul ${ }^{1,2}$, Chi-Hung Nguyen ${ }^{3}$, Jean-Noël Bastie ${ }^{1,2,4}$, Laurent Delva ${ }^{1,2}$, Ronan Quéré1,2

\author{
${ }^{1}$ Inserm UMR866, Université Bourgogne-Franche-Comté, \\ Faculté de médecine, 7, boulevard Jeanne d'Arc, \\ 21000 Dijon, France ; \\ ${ }^{2}$ LipSTIC Labex, 21000 Dijon, France ; \\ ${ }^{3}$ Institut Curie, UMR9187-U1196, CNRS-Institut Curie, Inserm, \\ Centre Universitaire, 91400 Orsay, France ; \\ ${ }^{4}$ Hôpital Universitaire François-Mitterrand, \\ Service d'Hématologie Clinique, 21000 Dijon, France. \\ laetitia.saintpaul@gmail.com \\ ronan.quere@inserm.fr
}

> L'hématopoïèse est un processus qui doit être finement régulé afin que chaque cellule sanguine mature soit produite en fonction des besoins physiologiques. Les cytokines participent à cette régulation en contrôlant l'auto-renouvellement, la survie, la prolifération et la différenciation des cellules hématopoïétiques. Selon leurs caractéristiques fonctionnelles et structurales, elles sont classées en sous-familles. Le GM-CSF (granulocyte/macrophage-colony stimulating factor) fait ainsi partie du groupe de cytokines qui agissent sur le lignage myéloïde. Il est produit au niveau de la moelle osseuse par les cellules du microenvironnement médullaire et certaines cellules hématopoïétiques [1]. La signalisation cellulaire induite par le GM-CSF active l'expression de gènes impliqués dans la survie et la prolifération cellulaire permettant ainsi aux cellules de se multiplier [2].

La voie du GM-CSF est suractivée dans les blastes leucémiques

Lorsque la régulation de l'hématopoïèse n'est plus assurée correctement, des proliférations affectant les lignages lymphoïde ou myéloïde peuvent se développer. Ces proliférations cellulaires conduisent à des leucémies lymphoblastiques ou myéloblastiques selon le type de cellules altérées. Les leucémies aiguës myéloblastiques (ou LAM) affectent les précurseurs de la lignée myéloïde qui se trouvent bloqués à un stade de leur différenciation. Les cellules immatures sont alors appelées blastes leucémiques. Elles prolifèrent de manière anormale et excessive et envahissent totalement la moelle osseuse, ce qui entraîne une inhibition de l'hématopoïèse normale et une insuffisance médullaire qui peut être fatale. L'une des particularités des blastes leucémiques est de présenter à leur surface le récepteur du GM-CSF alors qu'il n'est que faiblement exprimé dans les cellules souches hématopoïétiques (CSH) saines. Les blastes deviennent donc hypersensibles au GM-CSF, et la voie de signalisation dont il est à l'origine est activée de façon excessive. Le GM-CSF est impliqué dans l'activation de la survie et de la prolifération des cellules. L'hypersensibilité des blastes au GM-CSF pourrait donc contribuer à leur amplification au cours des leucémies [3]. Le potentiel thérapeutique d'une inhibition de la signalisation ayant pour origine le GM-CSF a été examiné ces dernières décennies et diverses stratégies ont été développées afin d'inhiber le récepteur du GM-CSF dans les blastes leucémiques $[4,5]$.

La voie de signalisation du GM-CSF est contrôlée selon la localisation de CD45 Le CD45 est un membre de la famille des protéines tyrosines phosphatases. II est exprimé par toutes les cellules hématopoiétiques nucléées. Dans les cellules myéloïdes, le CD45 déphosphoryle le site inhibiteur de la protéine kinase Lyn (au niveau de la tyrosine y507). La phosphorylation de ce site étant corrélée avec une inhibition de la signalisation du récepteur au GM-CSF [6-8], le CD45 contribue donc à activer la voie de signalisation induite par le GM-CSF. L'activité du CD45 est déterminée principalement par sa localisation par rapport à son substrat. Plus spécifiquement, le CD45 est plus ou moins actif selon qu'il soit localisé au niveau, ou en dehors, de microdomaines membranaires spécifiques appelés radeaux lipidiques ${ }^{l}$ [9]. Dans les cellules hématopoïétiques saines, le CD45 est localisé en dehors de ces radeaux lipidiques. II présente alors une faible activité. À l'inverse, dans les blastes leucémiques, le CD45 est situé au sein des radeaux lipidiques. II contribue alors à l'activation de la voie du GM-CSF et à la stimulation de la survie et de la prolifération des blastes leucémiques.

Pỵ entraîne l'exclusion du CD45 en dehors des radeaux lipidiques et inhibe la voie du GM-CSF dans les cellules leucémiques La pyrido[4,3-b] quinoxaline (PyQ) est un composé chimique provenant de la Chimiothèque nationale de l'institut

\footnotetext{
1 Un radeau lipidique ou raft lipidique est un microdomaine de la membrane plasmique, riche en sphingolipides, constituant un site privilégié pour l'activité de certaines protéines.
} 


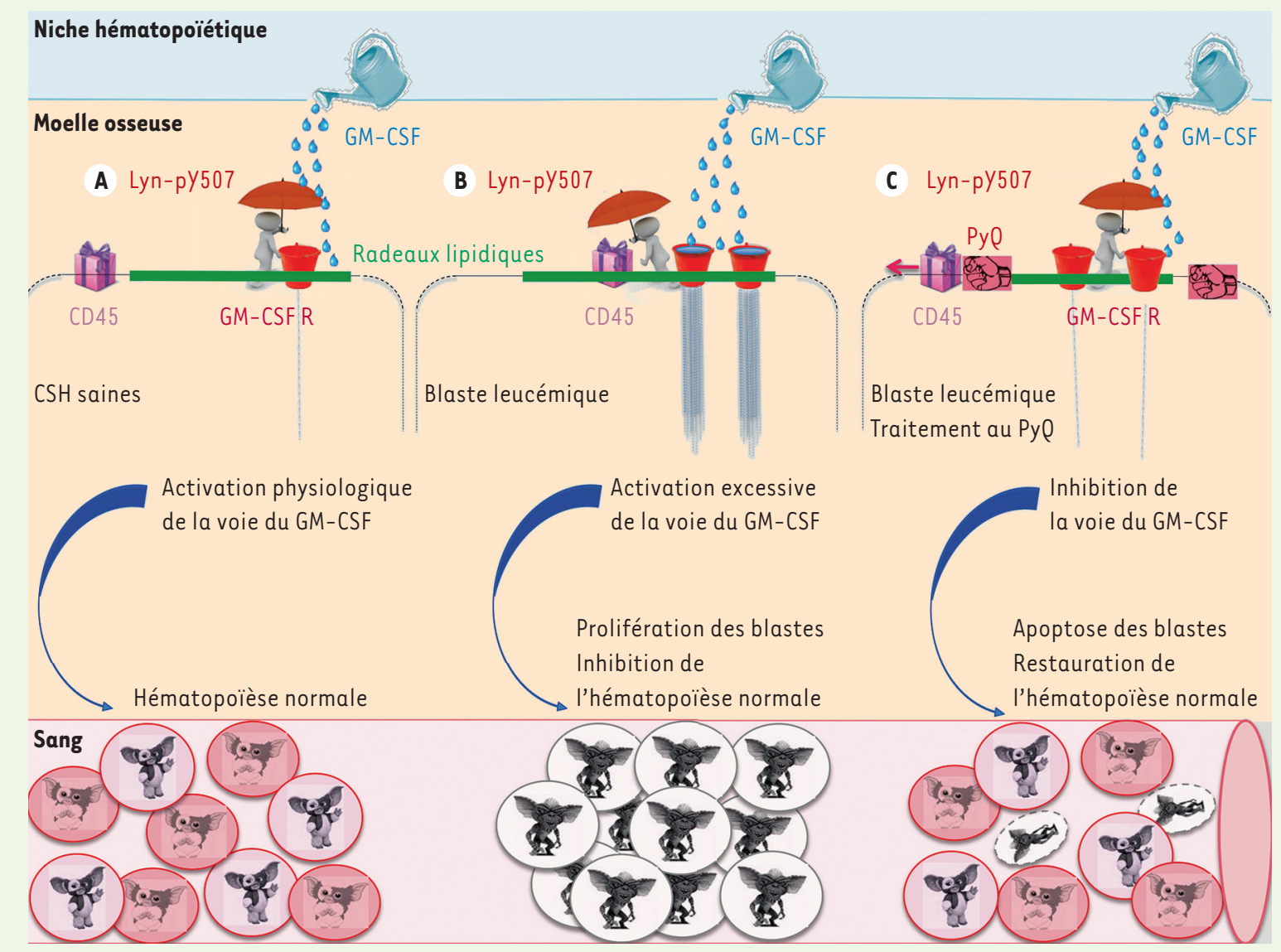

Figure 1. Pyrido[4,3-b]quinoxaline (Py) délocalise le CD45 en dehors des radeaux lipidiques et inhibe la voie du GM-CSF suractivée dans les blastes leucémiques. Le GM-CSF (granulocyte/macrophage-colony stimulating factor) est une cytokine produite par le microenvironnement médullaire activant des gènes de survie et de prolifération cellulaire. Cette voie de signalisation est régulée par les actions de Lyn, à activité kinase, et du CD45, à activité tyrosine phosphatase. Lorsqu'il est localisé dans les radeaux lipidiques, le CD45 déphosphoryle la tyrosine 507 de Lyn, la phosphorylation de cette tyrosine étant corrélée à une inhibition de la signalisation du GM-CSF. A. Dans des conditions physiologiques normales, le CD45 est regroupé en dehors des radeaux lipidiques, son activité tyrosine phosphatase est réduite. Lyn se trouve ainsi phosphorylé sur sa tyrosine 507, une tyrosine inactivante, qui va freiner l'activation du récepteur au GM-CSF. B. Dans les blastes leucémiques, le CD45 est localisé au niveau des radeaux lipidiques, ce qui lui permet de déphosphoryler le site inhibiteur de Lyn. La voie de signalisation du GM-CSF se trouve ainsi excessivement activée, ce qui provoque une prolifération anormale des blastes leucémiques. $C$. Un traitement par Py $\rho$ va exclure le CD45 en dehors des radeaux lipidiques, ce qui va entraîner la phosphorylation rapide du site inhibiteur de Lyn. La voie du GM-CSF est inhibée, ce qui bloque la prolifération des blastes leucémiques. Privés de leurs signaux de prolifération, l'apoptose des blastes leucémiques est induite, permettant une restauration de l'hématopoïèse normale. CSH : cellules souches hématopoïétiques.

Curie (UMR 176 CNRS, Institut Curie, Paris). Il s'agit d'un agent se fixant sur les membranes plasmiques. II pourrait aussi interférer avec les fonctions des récepteurs membranaires et, donc, leurs voies de signalisation. En utilisant des cellules de souris atteintes de LAM, nous avons montré qu'un traitement par PyQ permet de modifier la distribution membranaire du CD45 [10]. En effet, initialement localisé dans les radeaux lipidiques des blastes leucémiques, provenant de LAM humaines ou de cellules isolées d'un modèle murin de LAM, le CD45 est rapidement délocalisé après traitement par PyQ. Son activité phosphatase est alors profondément affectée, résultant en une augmentation rapide de la phosphorylation de Lyn sur son site inhibiteur et donc l'inhibition de la voie du GM-CSF (Figure 1), qui induit la mort des cellules leucémiques par blocage de leur prolifération qui dépend du GM-CSF.

Le CD45 n'est pas localisé dans les radeaux lipidiques dans les cellules saines. Py $\rho$ n'a donc aucun effet sur la régulation de la survie de ces cellules, d'où une faible toxicité observée sur les cellules hématopoïétiques primitives normales. Les blastes sont donc plus sensibles à Py $\rho$ que les CSH saines. $\varepsilon$ n effet, dans un modèle de souris 
développant une LAM, PyP permet de bloquer le développement leucémique. Les souris recevant deux injections de Py $\rho$ survivent significativement plus longtemps que le groupe de souris non traitées. Trois semaines après l'injection de PyQ, moins de $5 \%$ de blastes leucémiques sont retrouvés dans le sang des souris traitées et une reprise d'une hématopoï̀se normale est observée. À l'inverse, plus de $80 \%$ de blastes leucémiques sont retrouvés chez les souris non traitées qui succombent rapidement à la maladie. Py $Q$ augmente également significativement la survie de souris immunodéficientes xénogreffées avec des blastes leucémiques humains, par rapport aux souris non traitées qui développent plus rapidement une leucémie.
Py९ apparaît donc être une molécule d'intérêt avec un potentiel pharmacologique pour le traitement des LAM. $\diamond$ CD45 phosphatase, a relevant target for the treatment of acute myeloid leukemia

\section{LIENS D'INTÉRÊT}

Les auteurs déclarent n'avoir aucun lien d'intérêt concernant les données publiées dans cet article.

\section{RÉFÉRENCES}

1. Shearer WT, Rosenwasser LJ, Bochner BS, et al. Biology of common $\beta$ receptor-signaling cytokines: IL-3, IL-5, and GM-CSF. J Allergy Clin Immunol 2003 ; 112 : 653-65.

2. Hercus TR, Thomas D, Guthridge MA, et al. The granulocyte-macrophage colony-stimulating factor receptor: linking its structure to cell signaling and its role in disease. Blood 2009; 114 : 1289-98.

3. Birnbaum RA, O'Marcaigh A, Wardak Z, et al. Nfl and Gmcsf interact in myeloid leukemogenesis. Mol Cell $2000 ; 5: 189-95$.
4. Jakupovic I, Grandage VL, Linch DC, et al. Lack of effect of the human GM-CSF analog E21R on the survival of primary human acute myeloid leukemia cells. Blood $2004 ; 103$ : 3230-2.

5. Mathew M, Zaineb KC, Verma RS. GM-CSF-DFF40: a novel humanized immunotoxin induces apoptosis in acute myeloid leukemia cells. Apoptosis 2013; 18 : 882-95.

6. Hibbs ML, Harder KW. The duplicitous nature of the Lyn tyrosine kinase in growth factor signaling. Growth Factors $2006 ; 24: 137-49$.

7. Scapini P, Pereira S, Zhang H, et al. Multiple roles of Lyn kinase in myeloid cell signaling and function. Immunol Rev 2009 ; 228 : 23-40.

8. Wei S, Liu JH, Epling-Burnette PK, et al. Critical role of Lyn kinase in inhibition of neutrophil apoptosis by granulocyte-macrophage colony-stimulating factor. J Immunol 1996; 157 : 5155-62.

9. Simons K, Toomre D. Lipid rafts and signal transduction. Nat Rev Mol Cell Biol 2000 ; 1 31-9.

10. Saint-Paul L, Nguyen CH, Buffière A, et al. CD45 phosphatase is crucial for human and murine acute myeloid leukemia maintenance through its localization in lipid rafts. Oncotarget 2016; doi: 10.18632/oncotarget.11622.

\section{NOUVELle}

\section{L’antisynapse: présynapse et garde-fou de l'activation lymphocytaire?}

Chloé Guedj ${ }^{1}$, Nicolas Abraham, Clotilde Randriamampita
> Le lymphocyte $\mathrm{T}\left(\mathrm{L}_{\mathrm{T}}\right)$ est un acteur majeur de la réponse immunitaire adaptative. Son activation a lieu au sein des ganglions lorsqu'il reconnaît, à la surface d'une cellule présentatrice d'antigène (CPA) comme une cellule dendritique, l'antigène dont il est spécifique. L'activation des récepteurs à l'antigène (TCR) déclenche alors la relocalisation de nombreux éléments vers la zone de contact établie entre le $\mathrm{L}_{T}$ et la CPA, formant ainsi une structure appelée synapse immunologique. Cette plateforme de signalisation est composée de microagrégats, ou nanoclusters, organisés autour des TCR et à partir desquels sont déclenchés les signaux d'activation $[1](\rightarrow)$.

$(\rightarrow)$ Voir la Synthèse de J. Bouchet et

A. Alcover, $m / s n^{\circ}$ 6-7, juin-juillet 2014, page 665
On y retrouve de nombreuses protéines telles que les tyrosines kinases Lck (lymphocyte-specific protein tyrosine kinase) et ZAP-70 (70-kDa zetaassociated protein) ou la protéine adaptatrice LAT (linker for activation of $T$ cells). La formation de cette synapse immunologique est généralement perçue comme la première étape de l'activation lymphocytaire. Nos travaux ont cependant mis en évidence que, préalablement à la constitution de cette synapse, une structure transitoire, l'antisynapse, se formait au pôle opposé du contact entre $L_{T}$ et CPA $[2,3]$.

\section{L'antisynapse,}

un pôle de signalisation actif

Par des expériences de vidéo-microscopie et d'immunofluorescence, nous
Institut Cochin, Inserm U1016, CNRS UMR 8104, Université Paris-Descartes, Sorbonne Paris Cité, 22, rue Méchain,

75014 Paris, France.

${ }^{1}$ Adresse actuelle : Imagoseine, Institut Jacques Monod, Université Paris Diderot/CNRS, 15, rue Hélène Brion, 75205 Paris Cedex 13, France.

chloe.guedj@gmail.com

nicolas.abraham@inserm.fr

clotilde.randriamampita@inserm.fr

avons montré que tous les éléments classiquement décrits au sein de la synapse s'accumulaient également au niveau de l'antisynapse. C'est le cas par exemple des kinases Lck, ZAP-70, PI3K (phosphoinositide 3-kinase), de l'adaptateur LAT (Figure 1), mais aussi des radeaux lipidiques ${ }^{1}$ et des phospholipides PIP2 (phosphatidylinositol4,5-bisphosphate) et PIP3 (phosphatidylinositol-3,4,5-trisphosphate). Plus étonnant, la chaîne zeta du CD3 associé au TCR s'accumule également à ce pôle, mais cela de manière moins fréquente

\footnotetext{
1 Un radeau lipidique ou raft lipidique est un microdomaine de la membrane plasmique, riche en sphingolipides, constituant un site privilégié pour l'activité de certaines protéines.
} 